Rabaska

Revue d'ethnologie de l'Amérique française

\title{
Létourneau, Lorenzo. 17 Eldorado. Le Journal d'un chercheur d'or au Klondike, 1898-1902. Édité par François Gauthier. Montréal, Linguatech, 2006, 638 p. ISBN 2-920342-50-7.
}

\section{Jeanne Pomerleau}

Volume 5, 2007

URI : https://id.erudit.org/iderudit/019054ar

DOI : https://doi.org/10.7202/019054ar

Aller au sommaire du numéro

Éditeur(s)

Société québécoise d'ethnologie

ISSN

1703-7433 (imprimé)

1916-7350 (numérique)

Découvrir la revue

Citer ce compte rendu

Pomerleau, J. (2007). Compte rendu de [Létourneau, Lorenzo. 17 Eldorado. Le Journal d'un chercheur d'or au Klondike, 1898-1902. Édité par François

Gauthier. Montréal, Linguatech, 2006, 638 p. ISBN 2-920342-50-7.] Rabaska, 5,

176-178. https://doi.org/10.7202/019054ar d'utilisation que vous pouvez consulter en ligne.

https://apropos.erudit.org/fr/usagers/politique-dutilisation/ 
1938, le nouveau goût esthétique atteint le grand public avec des œuvres produites en série et commercialisées. Dans les années 1950, des artistes fondent le Retable et organisent ou participent à différentes expositions à Montréal, Rome, Québec, Washington... En 1952, une exposition provinciale sur l'art religieux lance un appel public aux artistes et artisans, des concours et des colloques sont organisés.

Ginette Laroche offre un regard éclairant sur le milieu de l'art religieux de l'époque. C'est une tranche de l'histoire de l'art québécois dont la richesse est à découvrir. Art sacré et matériaux nobles, art profane et matériaux populaires se méprisent, puis se côtoient et enfin dialoguent ; un style nouveau émerge alors que la hiérarchie académique des genres disparaît au profit d'un art plus démocratique et accessible. Cependant, le propos de l'auteur est parfois difficile à suivre alors que trop souvent des artistes ou des événements sont nommés sans être situés. À titre d'exemple, l'auteur affirme l'influence de Maurice Denis sur l'art de Borduas, mais sans expliquer comment elle se manifeste (p. 16). Le lecteur doit donc connaître à la fois les théories de l'art émergeant et la démarche créatrice des artistes nommés. De même, des œuvres non reproduites dans l'ouvrage sont citées pour appuyer son propos. Une connaissance préalable de ces œuvres est donc aussi requise. Dans l'ensemble, cet ouvrage n'en est pas un d'introduction et il vaut mieux connaître l'histoire de l'art religieux de cette époque pour y prendre plaisir. Heureusement pour les lecteurs qui voudraient poursuivre des recherches, l'auteur fait de nombreux renvois et donne des titres d'ouvrages spécialisés auxquels le néophyte peut se référer. De même, la sélection des œuvres reproduites permet d'appréhender les transformations et l'originalité des démarches artistiques durant la période à travers différents supports, matériaux et thèmes. Il s'agit donc d'un répertoire d'œuvres clés fort utile et d'un ouvrage à ranger dans sa bibliothèque.

Diane Joly

Université du Québec à Montréal

LÉTourneau, LoRenzo. 17 Eldorado. Le Journal d'un chercheur d'or au Klondike, 1898-1902. Édité par François Gauthier. Montréal, Linguatech, 2006, 638 p. ISBN 2-920342-50-7.

Lorenzo Létourneau de Saint-Constant de Napierville, qui partit le 9 janvier 1898 vers le Yukon, en vue d'atteindre le Klondike, tiendra un journal pendant tous les jours de sa vie à l'étranger, soit jusqu'au 28 août 1902. Ce 
journal est le plus complet, le plus intéressant et le plus représentatif de la vie des chercheurs d'or parmi ceux qu'il m'a été donné de consulter. Il est d'un grand intérêt, parce qu'on y retrouve aussi bien les difficultés rencontrées lors du voyage que celles vécues au Klondike, de même que les relations entretenues entre compagnons de travail. Létourneau, en faisant une description détaillée des activités techniques du métier de chercheur d'or, a aussi consigné le vocabulaire des chercheurs d'or canadiens-français.

Âgé de 31 ans au moment du départ, Létourneau qui avait fréquenté l'École normale Jacques-Cartier de Montréal, a une facilité d'écriture et il se chargera de faire la correspondance au nom de ses amis qui ne savent pas écrire. Presque toujours accompagné de compatriotes, dont plusieurs viennent de son village ou des alentours, il mettra un an et demi à se rendre au Klondike, où il demeurera ensuite jusqu'à son retour. Parti de Vancouver en bateau le 30 mars 1898, il descendit quelques jours plus tard à Wrangell, d'où il prit la route de Stickine, cherchant ensuite de l'or ici et là, sans succès, pendant seize mois avant d'arriver à Dawson le 8 juillet 1899 .

Il sera alors engagé par Narcisse Picotte, de Saint-Rémi de Napierville, qui possédait avec Jim Hall, un Américain, le claim 17 Eldorado et le claim 14 Bonanza, deux des plus riches concessions aurifères au Klondike. C'est là, avec les cinq frères Picotte et une trentaine d'autres travailleurs francophones, qu'il participera à tous les travaux exécutés par des chercheurs d'or et qu'il assistera aux premiers changements technologiques dans les mines du Klondike, comme l'installation des bouilloires pour dégeler le sol en hiver et l'arrivée des chars sur voies ferrées dans les corridors miniers souterrains. Létourneau, qui agit alors comme commis du claim et consigne les quantités d'or lavées chaque jour, voit défiler des cueillettes de quelque deux cent mille dollars.

Létourneau travaillera aussi avec Jack Tremblay, de Chicoutimi - d'abord contremaître sur le 17 Eldorado et plus tard propriétaire avec ses beauxfrères de la moitié du 14 Bonanza -, l'un des premiers chercheurs d'or canadiens-français. Tremblay, installé au Klondike avec son épouse en 1894, s'associera avec des mineurs australiens et américains qui s'en retournèrent riches dans leur pays à la fin de l'aventure.

Le journal de Létourneau nous apprend qu'il était toujours prêt à partir vers de nouveaux endroits où il entendait dire qu'il y avait de l'or. C'était là la caractéristique première d'un chercheur d'or. Il rapporte régulièrement les valeurs en or tirées de la terre par ses amis et les résultats sont importants. S'il s'est bien ennuyé de sa famille pendant les quatre ans et demi passés à l'étranger, il ne souhaitera pas moins en laissant le Klondike d'y « revenir un jour pas très éloigné ». À ses parents qui lui demandaient s'il était revenu riche du Klondike, il répondait : «Qu'est-ce que vous en pensez? » 
À son retour du Klondike, il entreprenait en 1903 une carrière de comptable qu'il exerça jusqu'en 1914 pour différentes entreprises, avant de devenir un des assistants d'Édouard-Zotique Massicotte aux Archives judiciaires de Montréal. Il cumulera aussi diverses fonctions comme celles de maire de Saint-Constant, préfet de comté de La Prairie, agent fédéral auprès des Amérindiens de Caughnawaga, secrétaire de la Commission scolaire et agent de la Banque canadienne nationale.

Cette publication comble un vide, car il existe peu de récits relatant « l'épopée des chercheurs d'or canadiens-français ». Les lecteurs seront choyés d'en apprendre autant sur le Klondike.

Jeanne Pomerleau

Québec

Maheu, Ange-Émile. Mon oncle Émile conte. Sudbury, Éditions Prise de parole, 2005, 282 p. + DC. ISBN 2-89423-173-3.

Le recueil d'Ange-Émile Maheu, Mon oncle Émile conte, appelle un double commentaire selon qu'on l'aborde du point de vue des genres narratifs ou de celui de la simple lecture dilettante.

En amont, la posture de l'auteur en regard de ses récits est triple. AngeÉmile Maheu endosse successivement plusieurs rôles. Il se fait tour à tour ethnographe, narrateur et auteur. Au cours de sa longue carrière de conteur et d'enseignant, il a archivé dans sa mémoire quantité de contes d'origine diverse - cela est visible dans l'énumération succincte qu'il dresse de ses sources dans le conte «Ti-Jean et la Vallée des âges » (p. 265) - de légendes, de croyances populaires et même d'un fait divers. Curieusement, cette historiette, pour reprendre une expression chère à $\mathrm{M}^{\mathrm{me}}$ de La Fayette, ne figure pas parmi les 45 titres recensés dans la table des matières. On la retrouve uniquement sur DC en cinquième position : «Le Docteur, le radium et le biscuit». À l'audition, le biscuit en question s'avère dans les faits un « beigne ». Ce récit n'est pas sans rappeler ces textes que publiait naguère Sélection du Reader's Digest : « L'être le plus extraordinaire que j'ai connu ».

L'ensemble des textes provient de l'enfance beauceronne de Maheu d'où le clin d'œil complice à Robert Cliche dans « La Réparation d'honneur » (p. 203) - de ses contacts avec les milieux traditionnels de transmission (camp de bûcherons dans les chantiers du Maine) et de ses lectures qu'on devine nombreuses : le Méphintos (p. 232) dans «Ti-Jean et les trois colombes » (p. 225) n'est pas sans évoquer le Méphistophélès du Faust de Gœthe. De l'aveu même de l'auteur anonyme de la « Présentation », bien qu'il puisse 